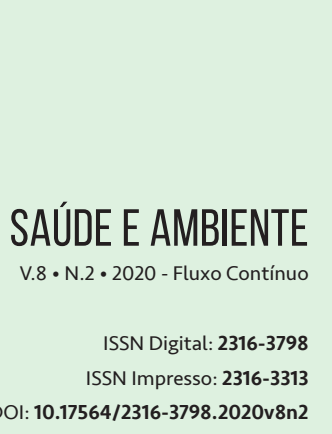

DOI: $10.17564 / 2316-3798.2020 v 8 n 2$

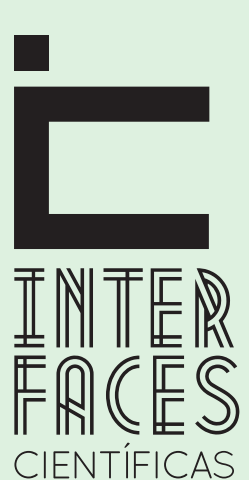

\section{PERFIL DOS ÓBITOS POR AGRESSÃO EM CRIANCGAS E ADOLESCENTES NO ESTADO DE SERGIPE ${ }^{1}$}

\section{PROFILE OF DEATHS FROM AGGRESSION IN CHILDREN AND ADOLESCENTS IN THE STATE OF SERGIPE}

\section{PERFIL DE MUERTES POR AGRESIÓN EN NIÑOS Y ADOLESCENTES ENEL ESTADO DE SERGIPE}

\title{
RESUMO
}

A violência contra crianças e adolescentes é um problema mundial de saúde pública. A cada 7 minutos, uma criança e adolescente é vítima de violência no mundo. Estudos internacionais indicaram que 91\% das mortes por armas de fogo de crianças entre 0 e 14 anos ocorrem nos Estados Unidos em relação aos outros países de renda alta. Sergipe, Rio Grande do Norte e Alagoas tiveram maior índice de violência em 2016 entre a população estudada. 0 objetivo deste estudo foi estabelecer o perfil sociodemográfico das vítimas de óbitos por agressão entre indivíduos de 0 a 18 anos no estado de Sergipe. Trata-se de um estudo transversal, de caráter descritivo e analítico, realizado por meio de coleta de dados nos 75 municípios do estado de Sergipe. As informações foram obtidas a partir do banco de dados do Ministério da Saúde, no Departamento de Informática do Sistema Único de Saúde e no Sistema de Informação sobre Mortalidade. Do total de registros de mortes de crianças e adolescentes entre 0 e 18 anos $(n=2.910)$, no recorte temporal de 2007 a 2016, foi observado que $60,1 \%$ das ocorrências acometeram adolescentes entre 17 e 18 anos, 92,4\% do sexo masculino, $87,3 \%$ cor/raça parda, $2 \%$ estado civil casado, com 4 a 7 anos de estudo, 53,0\% das agressões ocorreram na região de saúde Aracaju, 54,3\% no período noturno, sendo que $84,5 \%$ foram por arma de fogo. A agressão destaca-se com magnitude e prevalência de ocorrência dos casos de óbitos entre crianças e adolescentes, sendo que esse último grupo se apresenta como principal vítima.

\section{PALAVRAS-CHAVE}

1 Artigo baseado na dissertação de Mestrado de Fernanda Kelly Fraga Oliveira, intitulada Agressão como causa externa de óbitos em crianças e adolescentes no estado de Sergipe, defendida em 2019 no Programa de Pós-Graduação em Saúde e Ambiente da Universidade Tiradentes.

\author{
Kelly Fraga Oliveira ${ }^{2}$ \\ Henrique Soares Silva \\ Francisco Prado Reis ${ }^{5}$ \\ Íkaro Daniel de Carvalho Barreto ${ }^{7}$
}




\section{ABSTRACT}

Violence against children and adolescents is a worldwide public health problem. Every 7 minutes, a child and adolescent is a victim of violence in the world. International studies have indicated that $91 \%$ of firearm deaths in children between the ages of 0 and 14 occur in the United States compared to other high-income countries. Sergipe, Rio Grande do Norte and Alagoas had the highest rate of violence in 2016 among the population studied. The aim of this study was to establish the sociodemographic profile of victims of deaths due to aggression among individuals aged 0 to 18 years in the state of Sergipe. This is a cross-sectional, descriptive and analytical study, carried out through data collection in 75 municipalities in the state of Sergipe. The information was obtained from the database of the Ministry of Health, the Informatics Department of the Unified Health System and the Mortality Information System. Of the total records of deaths of children and adolescents between 0 and 18 years old $(n=2,910)$, in the time frame from 2007 to 2016, it was observed that $60.1 \%$ of the occurrences affected adolescents between 17 and 18 years old, $92.4 \%$ male, $87.3 \%$ color / brown race, $2 \%$ married marital status, with 4 to 7 years of study, $53.0 \%$ of the assaults occurred in the health region Aracaju, $54.3 \%$ at night, and $84.5 \%$ were by firearms. Aggression stands out with magnitude and prevalence of occurrence of deaths among children and adolescents, with the latter group presenting itself as the main victim.

\section{KEYWORDS}

Aggression; Mortality; Children; Adolescent.

\section{RESUMEN}

La violencia contra los niños y adolescentes es un problema mundial de salud pública. Cada 7 minutos, un niño y un adolescente son víctimas de la violencia en el mundo. Estudios internacionales han indicado que el $91 \%$ de las muertes por armas de fuego en niños entre 0 y 14 años ocurren en los Estados Unidos en comparación con otros países de altos ingresos. Sergipe, Rio Grande do Norte y Alagoas tuvieron la tasa más alta de violencia en 2016 entre la población estudiada. El objetivo de este estudio fue establecer el perfil sociodemográfico de las víctimas de muertes por agresión entre individuos de 0 a 18 años en el estado de Sergipe. Este es un estudio transversal, descriptivo y analítico, realizado a través de la recolección de datos en 75 municipios en el estado de Sergipe. La información se obtuvo de la base de datos del Ministerio de Salud, el Departamento de Informática del Sistema Único de Salud y el Sistema de Información de Mortalidad. Del total de registros de muertes de niños y adolescentes entre 0 y 18 años $(n=2,910)$, en el período de 2007 a 2016, se observó que el 
$60.1 \%$ de las ocurrencias afectaron a adolescentes entre 17 y 18 años, 92.4\% hombres, $87.3 \%$ color / raza marrón, $2 \%$ estado civil casado, con 4 a 7 años de estudio, $53.0 \%$ de los asaltos ocurridos en la región de salud Aracaju, 54.3\% en la noche y 84.5\% por armas de fuego. La agresión se destaca con la magnitud y la prevalencia de muertes entre niños y adolescentes, y este último grupo se presenta como la principal víctima.

\section{PALABRAS CLAVE}

Agresión; Mortalidad; Niño; Adolescente.

\section{INTRODUÇ̃̃̃O}

A violência afeta a saúde individual e coletiva, provoca mortes, lesões, traumas físicos e mentais, além de diminuir a qualidade de vida das pessoas e das comunidades (MINAYO et al., 2018). No tocante à criança e adolescente, a violência pode ser definida como todo ato ou omissão capaz de causar dano físico, sexual e/ou psicológico à vítima, seja ele cometido pelos pais ou parentes, seja por outras pessoas e instituições (MINAYO, 2001).

Segundo o Fundo das Nações Unidas para a Infância, a violência se expressa em quatro formas específicas: violência disciplinar e exposição à violência doméstica durante a primeira infância; violência na escola; mortes violentas de adolescentes; e violência sexual na infância e na adolescência (UNICEF, 2017).

Dentre os países de alta renda, cerca de $91 \%$ das mortes por armas de fogo de crianças de 0 a 14 anos ocorre nos Estados Unidos (EUA), o que tornam os ferimentos por armas de fogo (FAF) um grave problema de saúde pública nesse país, com as taxas mais elevadas de homicídios e suicídios por FAF. (GRINSHTEYN; HEMENWAY, 2016). Entre 2012 e 2014, uma média anual de 1.300 crianças morreram vítimas de FAF, resultando uma taxa anual de 1,8 por 100.000/hab. Os meninos, desproporcionalmente, suportaram o ônus da mortalidade por FAF, perfazendo cerca de $82 \%$ de todas as mortes em crianças (FOWLER et al., 2017).

Se, por um lado, a cada sete minutos uma criança ou adolescente entre 10 e 19 anos morre em consequência de violência no mundo (UNICEF, 2017), por outro lado, dados do Atlas da Violência elaborado pelo Instituto de Pesquisa Econômica Aplicada (IPEA) e pelo Fórum Brasileiro de Segurança Pública (FBSP), indicam um processo crescente da violência no Brasil. Em 2016, o país alcançou uma marca histórica de homicídios, segundo informações do Ministério da Saúde, ocorreram 30,3 mortes a cada 100 mil habitantes, o que corresponde a 30 vezes a taxa da Europa (CERQUEIRA et al., 2018).

No Brasil, a violência é classificada como estrutural, social e delinquencial. A primeira com expressões mais fortes no trabalho infantil e na existência de crianças, vivendo nas ruas e em instituições fechadas. A violência social se expressa mais fortemente em sua forma doméstica, enquanto a violência delinquencial tem as crianças tanto como vítimas quanto como atores (MINAYO, 2001). 
Os fatores que estimulam a violência letal no país são diversos e perpassam a densa desigualdade econômica e social entre crianças e adolescentes, a inoperância do sistema de segurança pública e a vigência de mercados ilícitos e facções criminosas. Tais fatores continuam desafiando governos e sociedade e persistem impulsionando a taxa de crimes letais, sobretudo no Norte e no Nordeste do Brasil. Apenas em 2016, houve um aumento do número de jovens assassinados, especialmente no Acre, Amapá, Rio de Janeiro, Bahia, Sergipe, Rio Grande do Norte, Alagoas e Roraima, com taxas ainda mais altas em Sergipe, Rio Grande do Norte e Alagoas (CERQUEIRA et al., 2018).

Além disso, a violência contra crianças e adolescentes inclui novos problemas para os serviços públicos e torna emergente o tema na área de conhecimento e de práticas do setor saúde. Assim, considerando a necessidade de uma atuação de prevenção e tratamento de base interdisciplinar, multiprofissional, intersetorial e socia (MINAYO et al., 2018), o presente estudo tem por objetivo estabelecer o perfil sociodemográfico das vítimas de óbitos por agressão entre indivíduos de 0 a 18 anos no estado de Sergipe, de 2007 a 2016.

\section{MÉTODO}

Trata-se de um transversal, de caráter descritivo e analítico, realizado por meio de coleta de dados secundários, sendo a população de estudo composta pela quantidade de criança e adolescente/ano. Seguindo todos os aspectos éticos preconizados pela Resolução: $n^{0}$ 466/2012 do Conselho Nacional de Saúde e pelo Comitê de Ética em Pesquisa, em aprovação CAAE nº 70648317.2.0000.5371 e Parecer n 2.435.980.

0 presente estudo foi realizado em todos os municípios Sergipanos, totalizado 75 municípios. De acordo com o Instituto Brasileiro de Geografia e Estatística (IBGE), o estado de Sergipe apresenta como fronteiras a Leste o Oceano Atlântico, a Oeste e ao Sul o estado da Bahia e ao Norte o estado de Alagoas, é formado por três mesorregiões geográficas, que são: Agreste Sergipano, Leste Sergipano e Sertão Sergipano e essas subdividem-se em treze microrregiões denominados: Agreste de Itabaiana, Agreste de Lagarto, Aracaju, Baixo Cotinguiba, Boquim, Carira, Cotinguiba, Estância, Japaratuba, Nossa Senhora das Dores, Propriá, Sergipana do Sertão do São Francisco e Tobias Barreto, contando ainda com sete regiões de saúde: Aracaju, Estância, Itabaiana, Lagarto, Nossa Senhora da Glória, Nossa Senhora do Socorro e Propriá (IBGE, 2015).

A população específica compreendeu crianças e adolescentes de 0 a 18 anos completos, 18 anos 11 meses e 29 dias, vítimas de mortalidade por agressão, sendo excluídas aquelas que já completaram 19 anos, utilizando as categorias segundo a Organização Mundial da Saúde e Ministério da Saúde, e divididas em ocorrências de 0 a 14 anos, de 15 a 16 anos e de 17 a 18 anos e sexo (OPAS/OMS, 2018).

Após aprovação do Comitê de Ética em Pesquisa, foi prosseguida a coleta dos dados de mortalidade relativos ao período de 2007 a 2016. As informações foram obtidas a partir do banco de dados do Ministério da Saúde, no Departamento de Informática do Sistema Único de Saúde (DATASUS) e no Sistema de Informação sobre Mortalidade (SIM), para particularizar as ocorrências de mortalidade por agressão nos 75 municípios sergipanos; bem como, no IBGE para obtenção de contagem popula- 
cional entre o período estudado (BRASIL, 2010; IBGE, 2015). Os dados suplementares foram trabalhados segundo a tipificação das mortes por agressão, de acordo com a Classificação Internacional de Doença (CID-10) da Organização Mundial da Saúde e Ministério da Saúde (OPAS/OMS, 2018).

Os dados foram descritos por meio de frequências absoluta e relativa. Testamos a hipótese de independência entre as variáveis categóricas por meio do teste Qui-Quadrado de Pearson. As comparações múltiplas para proporção foram testadas por meio do teste $Z$ com correção de Bonferroni. Também foi realizada análise exploratória das variáveis e para verificar possíveis associações utilizou-se a análise de correspondência múltipla.

As associações entre as variáveis foram representadas graficamente em mapa perceptual, organizada em linhas e colunas e a proximidade expressa pelas variáveis indicam o grau de associação entre elas. Foram incluídas variáveis significativas a $5 \%$ pelo teste Qui-Quadrado com as regiões de saúde de residência e ocorrência. As análises foram realizadas utilizando o software R, versão 3.4.0 (R CORE TEAM, 2016) e o nível de significância adotado foi de $5 \%$.

\section{RESULTADOS}

Na faixa etária de 17 a 18 anos observou-se 60,1\% das ocorrências, com predomínio do sexo masculino $(92,4 \%)$, de cor/raça parda $(87,3 \%)$, estado civil solteiro $(99,7 \%)$ e que possuíam de 4 a 7 anos de estudo (59,3\%), a maioria residia na região de saúde de Aracaju (45,1\%), onde ocorreram $53,0 \%$ das agressões. As agressões ocorreram no período noturno $(54,3 \%)$ por meio de arma de fogo (84,5\%). Observou-se que três $(0,3 \%)$ adolescentes tinham estado civil casado ou separado judicialmente/divorciado.

Quanto às $45(4,1 \%)$ crianças (0-11 anos), quando associada região de saúde de ocorrência e residência com sexo, raça/cor, turno de ocorrência e causa base foi observado diferenças significativas apenas entre as regiões de Aracaju e Propriá, onde 9 (75\%) crianças residentes em Aracaju e $13(68,4 \%)$ que morreram nessa região tiveram causa base de arma de fogo enquanto a região de Propriá não reportou nenhum caso de residente morto por arma de fogo nem cuja ocorrência se deu nesta região.

Quanto às variáveis sociodemográficas dos adolescentes vítimas de agressão no período de 2007 a 2016 no estado de Sergipe divididos por região de saúde da ocorrência estão representadas na Tabela 1. Não foram observadas diferenças significativas quando se associou região de saúde de ocorrência e sexo, raça/cor e turno da ocorrência. Observou-se diferenças significativas quanto a escolaridade e causa base.

Quanto a prevalência de adolescentes cuja escolaridade era nenhuma, a região de ocorrência de Aracaju (0,9\%) tinha prevalência inferior as regiões de ocorrência de Glória (8\%) e Lagarto (6,2\%). Quanto a causa base por meio de objeto cortante ou penetrante, as regiões de ocorrência de Aracaju $(10,2 \%)$ e Estância $(21,6 \%)$ diferiram. 
Tabela 1 - Variáveis sociodemográficas - adolescentes vítimas de agressão e Região de Saúde de Ocorrência em Sergipe/Brasil, 2007 -2016

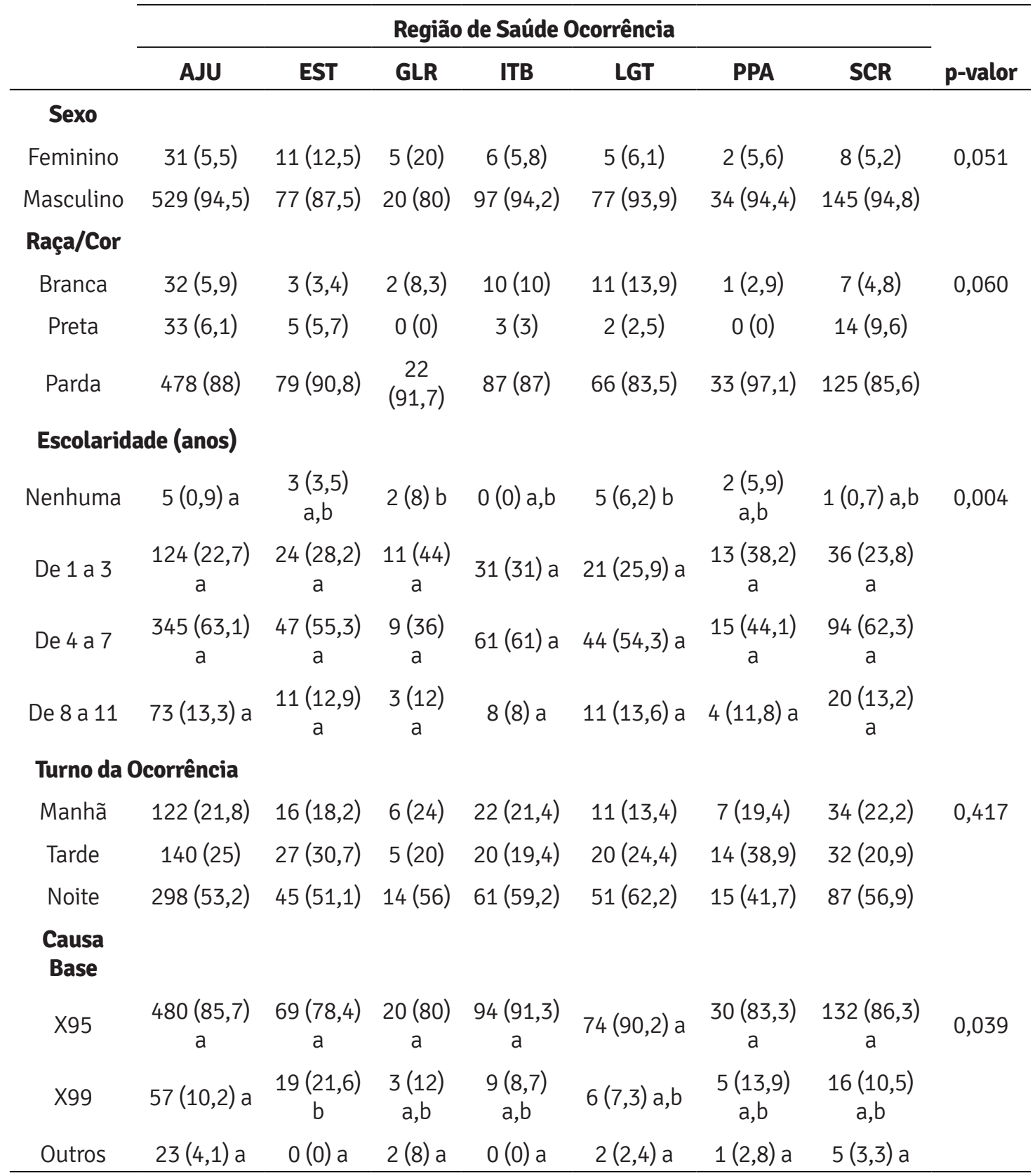

Legenda: N - Frequência absoluta; \% - Frequência relativa percentual; Teste Qui-Quadrado de Pear- 
son; a,b,c Subgrupos distintos para o teste Z para proporções com correção de Bonferroni; AJU - Aracaju; EST - Estância; GLR - Nossa Senhora da Glória; ITB - Itabaiana; LGT - Lagarto; PPA - Propriá; SCR - Nossa Senhora do Socorro; Separado - Separado Judicialmente/Divorciado; X95 - Agressão por meio de disparo de arma de fogo; X99 - Agressão por meio de objeto cortante ou penetrante.

Fonte: Autores.

Para uma melhor interpretação do perfil das crianças e adolescentes vítimas de agressão, foi construído um mapa perceptual estimado por meio de análise de correspondência múltipla apresentado na Figura 1. Na dimensão 1, as categorias mais distantes da origem são Glória, Propriá e Estância e na dimensão 2 as regiões de Glória, Itabaiana e Estância, o que indica que as regiões de Glória e Estância são as que mais se distanciam da hipótese de independência. Assim, foi possível identificar possíveis associações entre a região de residência Estância e a causa base X99 e a região de Glória e nenhuma escolaridade devido a estas classes estarem no mesmo quadrante, estarem mais distantes da origem e possuírem um ângulo pequeno entre si em relação a origem (SOURIAL et al., 2010).

Figura 1 - Mapa perceptual estimado por Análise de Correspondência Múltipla para Causa-Base, Região de Saúde de Residência e Escolaridade, 2007 a 2016

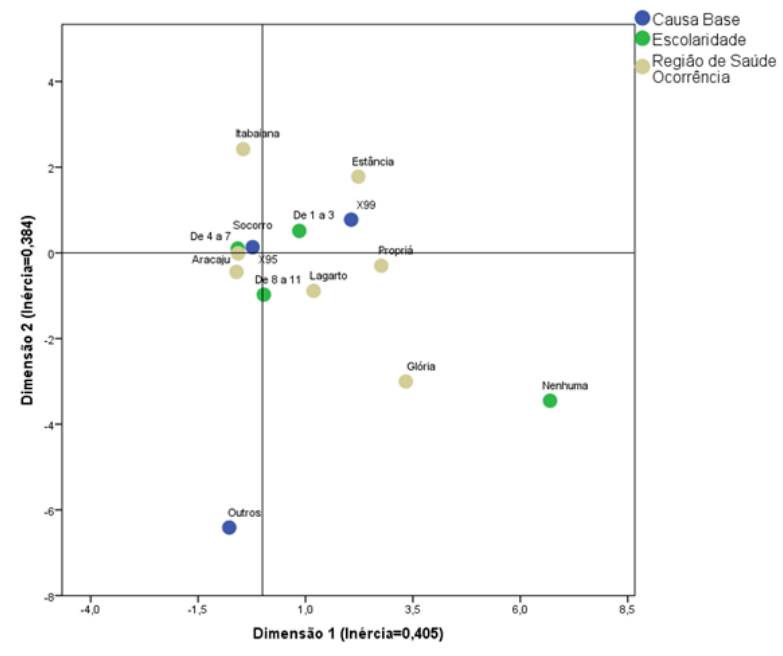

Fonte: Autores.

Quanto as variáveis sociodemográficas dos adolescentes vítimas de agressão no período de 2007 a 2016 no estado de Sergipe divididos por região de saúde da residência estão representadas na Tabela 2. Não foram observadas diferenças significativas quando se associou região de saúde de residência e turno da ocorrência. Observou-se diferenças significativas quanto ao sexo, raça/cor, escolaridade e causa base. 
Evidencia-se que os adolescentes do sexo masculino são mais prevalentes em Aracaju (95,2\%) do que a região de Glória (81,3\%); a raça/cor branca foi mais observada na região de Lagarto (16,3\%) do que as regiões de Aracaju (4,9\%) e Socorro (3,6\%); adolescentes com nenhuma escolaridade foram mais observados na região de Lagarto $(5,7 \%)$ do que em Aracaju (0,9\%); adolescentes com escolaridade entre 1 e 3 anos foram mais observados na região de Glória (46,9\%) do que em Aracaju (22,3\%); adolescentes com escolaridade entre 4 e 7 anos foram mais observados na região de Socorro (66,3\%) do que em Glória (37,5\%); quanto a causa base de agressão por meio de objeto cortante ou penetrante na região de Estância (24,2\%) foi mais observada do que nas regiões de Aracaju (10\%) e Socorro (8,9\%).

Tabela 2 - Variáveis sociodemográficas - adolescentes vítimas de agressão e Região de Saúde de Residência em Sergipe/Brasil, 2007 a 2016

Região de Saúde Ocorrência

\begin{tabular}{|c|c|c|c|c|c|c|c|c|}
\hline & AJU & EST & GLR & ITB & LGT & PPA & SCR & p-valor \\
\hline \multicolumn{9}{|l|}{ Sexo } \\
\hline Feminino & $23(4,8) a$ & $\begin{array}{c}11(12,1) \\
a, b\end{array}$ & $\begin{array}{c}6(18,8) \\
b\end{array}$ & $8(7) a, b$ & $8(9) a, b$ & $\begin{array}{c}2(5,4) \\
a, b\end{array}$ & $\begin{array}{c}10(4,9) \\
a, b\end{array}$ & 0,012 \\
\hline Masculino & $\begin{array}{c}458 \\
(95,2) a\end{array}$ & $\begin{array}{c}80(87,9) \\
\text { a,b }\end{array}$ & $\begin{array}{c}26(81,3) \\
b\end{array}$ & $\begin{array}{c}106(93) \\
a, b\end{array}$ & $\begin{array}{c}81(91) \\
a, b\end{array}$ & $\begin{array}{c}35(94,6) \\
a, b\end{array}$ & $\begin{array}{c}193(95,1) \\
a, b\end{array}$ & \\
\hline \multicolumn{9}{|l|}{ Raça/Cor } \\
\hline Branca & $23(4,9) a$ & $\begin{array}{c}5(5,6) \\
a, b\end{array}$ & $\begin{array}{c}2(6,9) \\
a, b\end{array}$ & $\begin{array}{c}12(11) \\
a, b\end{array}$ & $\begin{array}{c}14(16,3) \\
b\end{array}$ & $\begin{array}{c}3(8,6) \\
a, b\end{array}$ & $7(3,6) \mathrm{a}$ & 0,005 \\
\hline Preta & $31(6,6) \mathrm{a}$ & $5(5,6) \mathrm{a}$ & $0(0) a$ & $2(1,8) \mathrm{a}$ & $2(2,3) \mathrm{a}$ & $2(5,7) \mathrm{a}$ & $15(7,7) \mathrm{a}$ & \\
\hline Parda & $\begin{array}{c}414 \\
(88,5) a\end{array}$ & $\begin{array}{c}80(88,9) \\
\mathrm{a}\end{array}$ & $\begin{array}{c}27(93,1) \\
a\end{array}$ & $\begin{array}{c}95(87,2) \\
a\end{array}$ & $\begin{array}{c}70(81,4) \\
a\end{array}$ & $\begin{array}{c}30(85,7) \\
a\end{array}$ & $\begin{array}{c}174(88,8) \\
a\end{array}$ & \\
\hline
\end{tabular}

\section{Escolaridade (anos)}

$\begin{array}{ccccccccc}\text { Nenhuma } & 4(0,9) a & 3(3,4) & 2(6,3) & 0(0) a, b & 5(5,7) b & 2(5,7) & 2(1) a, b & 0,001 \\ & & a, b & a, b & a, b & & \\ \text { De 1 a 3 } & 105 & 27(30,7) & 15(46,9) & 34(30,6) & 22(25) & 12(34,3) & 45(22,6) & \\ & (22,3) a & a, b & b & a, b & a, b & a, b & a, b \\ \text { De 4 a 7 } & 289 & 47(53,4) & 12(37,5) & 67(60,4) & 49(55,7) & 19(54,3) & 132(66,3) \\ & (61,5) a, b & a, b & b & a, b & a, b & a, b & a \\ \text { De 8 a 11 } & 72(15,3) & 11(12,5) & 3(9,4) a & 10(9) a & 12(13,6) & 2(5,7) a & 20(10,1) \\ & a & a & & a & a & \end{array}$


Região de Saúde Ocorrência

$\begin{array}{lllllll}\text { AJU EST } & \text { GLR } & \text { ITB } & \text { LGT } & \text { PPA } & \text { SCR } & \text { p-valor }\end{array}$

\section{Turno da Ocorrência}

$\begin{array}{ccccccccc}\text { Manhã } & 105 & 15(16,5) & 8(25) & 23(20,2) & 12(13,5) & 8(21,6) & 47(23,2) & 0,170 \\ & (21,8) & & & & & & \\ \text { Tarde } & 127 & 30(33) & 5(15,6) & 22(19,3) & 23(25,8) & 12(32,4) & 39(19,2) \\ & (26,4) & & & & & & \\ \text { Noite } & 249 & 46(50,5) & 19(59,4) & 69(60,5) & 54(60,7) & 17(45,9) & 117(57,6)\end{array}$

\section{Causa Base}

\begin{tabular}{|c|c|c|c|c|c|c|c|c|}
\hline X95 & $\begin{array}{c}416 \\
(86,5) \mathrm{a}\end{array}$ & $\begin{array}{c}68(74,7) \\
a\end{array}$ & $\begin{array}{c}27(84,4) \\
a\end{array}$ & $\begin{array}{c}103 \\
(90,4) a\end{array}$ & $\begin{array}{c}78(87,6) \\
a\end{array}$ & $\begin{array}{c}30(81,1) \\
a\end{array}$ & $\begin{array}{c}177(87,2) \\
a\end{array}$ & 0,022 \\
\hline 99 & $48(10) \mathrm{a}$ & $\begin{array}{c}22(24,2) \\
b\end{array}$ & $\begin{array}{c}3(9,4) \\
a, b\end{array}$ & $\begin{array}{c}10(8,8) \\
a, b\end{array}$ & 8 (9) a,b & $\begin{array}{c}6(16,2) \\
a, b\end{array}$ & $18(8,9) \mathrm{a}$ & \\
\hline
\end{tabular}

Outros $\quad 17(3,5)$ a $\quad 1(1,1) a \quad 2(6,3)$ a $\quad 1(0,9)$ a $\quad 3(3,4)$ a $\quad 1(2,7)$ a $\quad 8(3,9) a$

Legenda: N - Frequência absoluta; \% - Frequência relativa percentual; Teste Qui-Quadrado de Pearson; a,b,c Subgrupos distintos para o teste Z para proporções com correção de Bonferroni; AJU - Aracaju; EST - Estância; GLR - Nossa Senhora da Glória; ITB - Itabaiana; LGT - Lagarto; PPA - Propriá; SCR - Nossa Senhora do Socorro; Separado - Separado Judicialmente/Divorciado; X95 - Agressão por meio de disparo de arma de fogo; X99 - Agressão por meio de objeto cortante ou penetrante. Fonte: Autores.

Para visualizar o perfil dos adolescentes na comparação com a região de residência, foi construído um mapa perceptual estimado por meio de análise de correspondência múltipla apresentado na Figura 2.

Na dimensão 1, as regiões de residência mais distantes da origem são Glória, Itabaiana, Lagarto e Propriá e na dimensão 2 as regiões de Glória, Estância e Lagarto, o que indica que as regiões de Glória e Lagarto são as que mais se distanciam da hipótese de independência. Pode-se destacar associações entre a região de residência de Estância e a causa base X99 além de Lagarto e a raça/cor branca devido a estas classes estarem no mesmo quadrante, estarem mais distantes da origem e possuírem um ângulo pequeno entre si em relação a origem (SOURIAL et al., 2010). 
Figura 2 - Mapa perceptual estimado por Análise de Correspondência Múltipla para Causa-Base, Escolaridade, Raça/Cor, Sexo e Região de Saúde de Residência, 2007 a 2016

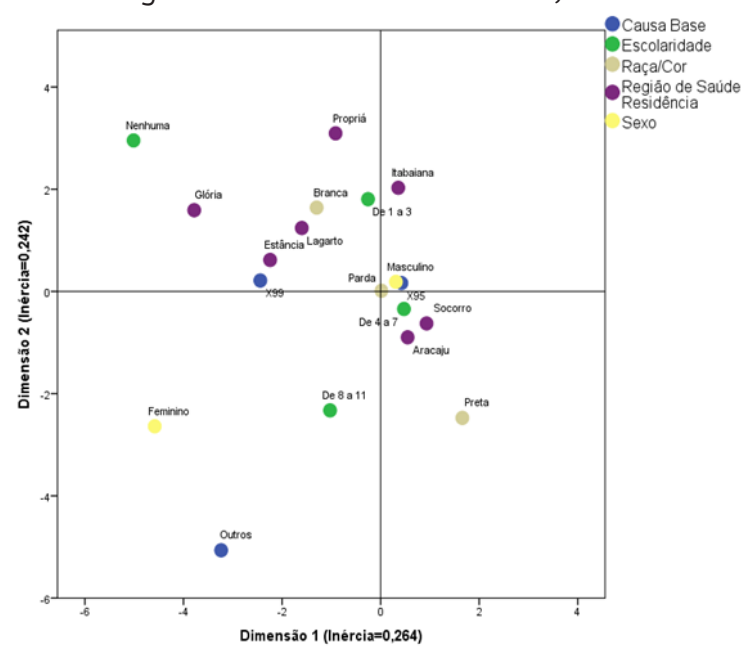

Fonte: Autores.

\section{DISCUSSÃO}

Estudos contemporâneos, tendo em face a violência como destaque no mundo, reconhecem que este é um problema mundial, multifatorial, em ascendência, embora com diversas intensidades e causas conforme a área geográfica, acomentendo todas as classes sociais e culturais, conquanto a literatura científica retrata e evidencia a temática com a realidade da magnitude do problema, visto que, as causas externas de mortalidade podem ser previsíveis e preveníveis.

A violência no Brasil se configura em um problema de saúde pública de grande proporção e transcendência social e cultural, que tem provocado grande impacto de relevância pública devido a causas externas de morbimortalidade. As estatísticas de mortalidade são muito úteis como fonte de informação para avaliar as condições de saúde da população, contudo, analisando esses dados é possível refletir que a estruturação familiar e a gestão pública possivelmente contribuem com a evolução dessa violência.

Observou-se neste estudo, dentre as variáveis sociodemográficas das crianças e adolescentes no período analisado, que a faixa etária entre 17-18 anos compreendeu a maioria das ocorrências, predominando o sexo masculino, estado civil solteiro, com 4 a 7 anos de estudo, onde as agressões ocorreram à noite por arma de fogo na região de saúde Itabaiana. Esses dados corroboram com estudo realizado em Manaus por Orellana e colaboradores (2017) que evidenciaram a influência do estado civil dos indivíduos no cenário avaliado, onde as chances de homicídios foram maior em solteiros quando comparado com os casados, assim como, aumento das agressões em jovens com pouca escolaridade. 
Ainda, Cunningham e colaboradores (2015) revelaram que no setor de trauma pediátrico em hospital de Michigan-EUA, as lesões relacionadas a agressão entre adolescentes é uma das principais causas de morbimortalidade por violência, como também, o risco de lesões relacionadas à agressão, o homicídio tem sido a principal causa de morte entre adolescentes e jovens do sexo masculino.

Ao analisar faixa etária e sexo, raça/cor e causa base, foi possível identificar associação estatística significante entre as variáveis estudadas, com a maioria das ocorrências por agressões por meio de arma de fogo que acometeram adolescentes entre 15 e 18 anos. Contudo, autores identificaram que o perfil do jovem vítima de homicídio, é do sexo masculino e que Sergipe segue com a taxa mais elevada 280,6 por um grupo de 100 mil habitantes (CERQUEIRA et al., 2018).

As taxas de homicídios dos EUA foram sete vezes mais altas do que em outros países de alta renda, impulsionadas por uma taxa de homicídios por arma de fogo que foi 25,2 vezes maior. Para os jovens de 15 a 24 anos, a taxa de homicídios por armas de fogo foi 49,0 vezes maior (GRINSHTEYN; HEMENWAY, 2016).

A taxa anual de morte por arma de fogo para meninos foi 4,5 vezes maior que a taxa anual para meninas, em estudo realizado entre 2012 e 2014, essa diferença foi ainda mais pronunciada pela idade, com a taxa de meninos de 13 a 17 anos, sendo 6 vezes mais alta do que a taxa para meninas de mesma idade (FOWLER et al., 2017). Estudo realizado por Camargo e colaboradores (2018) revelou que a maior parte das vítimas por arma de fogo foram os homens, com idade do óbito em média de 30 anos, com potencial de vida perdida entre variação percentual positiva e evolução temporal dos óbitos.

Entre 1980 e 2016 cerca de 910 mil pessoas foram mortas por perfuração de arma de fogo no país, um aumento de proporção crescente aconteceu desde meados de 1980, para tanto, em 2003 foi sancionado o Estatuto do Desarmamento, a proporção de homicídios com o uso de armas de fogo que crescia ininterruptamente de 40\% a 71,1\%, ficando estável de 2003 a 2016 (CERQUEIRA et al., 2018).

A exposição dos homens a fatores de riscos individuais, como o consumo de álcool, fumo e outras drogas, além do uso de arma de fogo e maior inserção no mercado informal. Em um estudo realizado por Minayo (1997), sobre a exposição a riscos e vulnerabilidade social dessa população, foi observado como fator relevante o predomínio do sexo masculino nas principais ocorrências por mortes violentas no Brasil, sendo o facilitador dessas ocorrências o aumento de trabalho, principalmente em atividades ilícitas.

As autoridades sergipanas acreditam haver relação entre os homicídios de todas as idades e o uso ou tráfico de drogas em vários municípios (FONSECA et al., 2014). Matos e Martins (2012) discutem ainda que, a populção de jovens do sexo masculino está mais susceptível às causas externas de mortalidade, devido à imaturidade, espiríto de aventura, excesso de coragem e uso de álcool e drogas.

Estudo realizado por Camargo e colaboradores (2018) aponta que dentre os diferentes fatores que contribuíram para as vítimas de óbito por agressão estão o desemprego e a segurança pública. Embora autores indiquem que as altas taxas de homicídios e suicídios por arma de fogo aumentaram em países de alta renda, enquanto as taxas de mortes por arma de fogo diminuíram em outros países (GRINSHTEYN; HEMENWAY, 2016).

A análise das variáveis por região de saúde de residência, apresentou associações entre região de saúde de residência, sexo, raça/cor, escolaridade e causa base. A região de Glória teve maior índice 
das ocorrências no sexo feminino, a cor/raça branca sobressaiu nas ocorrências da região de Lagarto, com menor escolaridade na região de Glória apresentou menor tempo de escolaridade nas duas variáveis: de 4 a 7 anos e de 1 a 3 anos de estudo. Existe uma relação com o grau de escolaridade e a violência evidenciado neste estudo, corroborando com estudo de Orellana e colaboradores (2017) que revelam que entre as vítimas com maior nível de escolaridade, a probabilidade predita de homicídio é bem inferior naquelas com menor nível de escolaridade, que sobretudo, estão mais expostas.

Quando analisada a violência letal contra adolescentes, acentua-se uma situação mais grave, os homicídios, correspondem a maioria da causa de óbito de homens entre 15 e 19 anos. Cerqueira e colaboradores (2018) reforçam que a juventude perdida se trata de um problema de importância no caminho do desenvolvimento social do país e quem vem aumentando diariamente. Tal resultado, compreende a semelhança deste estudo, onde a maior vulnerabilidade foi identificada nos homens, esses agravos podem estar atrelados, em sua maioria a um processo cultural que se inicia na infância, quando há maior liberdade aos meninos, enquanto para as meninas há uma maior vigilância.

Ao identificar à causa base das agressões, a região de Itabaiana apresentou maior prevalência das ocorrências por arma de fogo, seguida das regiões de Aracaju e Propriá. Lesões por arma de fogo é um importante problema de saúde pública, contribuindo substancialmente para a morte prematura e incapacidade das crianças. Entender sua natureza e impacto é um primeiro passo em direção à prevenção. As lesões por arma de fogo estão entre as mais altas taxas de fatalidade (FOWLER et al., 2017). Fiscalizar e controlar o uso da arma de fogo é inevitável, pois em situações de crescimento da violência letal contra crianças e adolescentes na última década, a associação concomitantemente, é evidenciada pelo crescimento de vítimas por arma de fogo.

O perfil das variáveis sociodemográficas das crianças e adolescentes vítimas de agressão, verificado nas regiões de residência e de ocorrência, aponta associação significativa entre raça/ cor, escolaridade, sexo e causa/base, o que coincide com outros estudos em que a mortalidade por causas externas de adolescentes é decorrente de agressão por arma de fogo, acometendo jovens do sexo masculino e com menor grau de instrução. Um dos fatores dos acontecimentos provavelmente é o envolvimento dos jovens em atividades ilegais como o tráfico, uso de drogas ilícitas e acesso facilitado a arma de fogo.

Para tanto Matos e Martins (2012) descreve que, as causas externas de mortalidade no país foram os primeiros motivos de óbito em grandes números, compreendendo a faixa etária entre 0 e 24 anos, apresentando como principal fator que favorece a susceptibilidade dos acontecimentos, o processo desestruturado de urbanização, o aumento da desigualdade social e a exclusão da população de baixa renda, eventos que contribuem para o aumento da violência urbana.

A comparação entre as categorias e associação entre residência e ocorrência nas regiões de saúde por agressão mostrou-se expressiva entre as regiões de Aracaju e Nossa Senhora do Socorro, cor/ raça parda e vítimas de disparo por arma de fogo. Ocorrência apontada por estudo realizado em 2012, que expõe sobre a violência contra crianças e adolescentes em jornais de Sergipe, dentre 698 fatos noticiados, $46 \%$ das notícias referiam-se a região metropolitana de Aracaju, seguida dos municípios de Nossa Senhora do Socorro e Itabaiana (ANSELMO, 2014). 
A pesquisa realizada trouxe limitações importantes quanto ao estudo observacional e a completude dos dados analisados. A completude limitou a evidência de informações consideradas pertinentes na elucidação dos casos. Outra limitação do estudo é a base populacional estimada de crianças e adolescentes pelo IBGE de 2010, onde foram analisados os casos ocorridos entre 2007 e 2016.

\section{CONCLUSÃO}

Ao estabelecer o perfil sociodemográfico das vítimas de óbitos por agressão entre indivíduos de 0 a 18 anos no estado de Sergipe, do total de registros de mortes de crianças e adolescentes no recorte temporal de 2007 a 2016, foi observado que a maioria das evidências acometeram adolescentes entre 17 e 18 anos, do sexo masculino, cor/raça parda, estado civil casado, com 4 a 7 anos de estudo, o maior número das agressões ocorreram na região de saúde Aracaju, no período noturno, prevalecendo ocorrências por arma de fogo.

O impacto econômico da mortalidade por agressão em crianças e adolescentes no estado, pode ser medido diretamente por meio da vitimização por causas externas dos adolescentes com resultados estatísticos significativos, principalmente para a mortalidade por agressão. É imprescindivel a execução de estratégias e ações em defesa da criança e do adolescente, exprimidas por políticas públicas e fortalecimento da rede de atenção a proteção e cuidado.

Torna-se imperioso, tomando como base o número de óbitos classificados pela Classificação Internacional de Doenças (CID-10) como eventos que envolvem agressões e óbitos provocados por intervenção legal, investir numa disposição institucional que habilite o estado e the garanta os instrumentos de governo para que se possa efetivamente implementar políticas que nos levem a um estado com mais paz no futuro.

Este trabalho poderá contribuir com futuros estudos e desenvolvimento de pesquisas acerca do tema abordado, visando estabelecer o impacto causado na sociedade, identificando a agressão como um problema de saúde pública. Além disso, poderá contribuir para o estado e municípios da pesquisa, numa perspectiva de fortalecer e implementar estratégias de prevenção, acolhimento e intervenção indispensáveis para minimizar o problema.

\section{REFERÊNCIAS}

ANSELMO, R. Lentes de papel: ensaio sobre as notícias de violência contra crianças e adolescentes em Sergipe no ano de 2012. Aracaju: Instituto Braços - Centro de Defesa dos Direitos Humanos em Sergipe, 2014.

BRASIL. Ministério da Saúde. DATASUS. Informações de saúde. Estatísticas de mortalidade: óbitos por ocorrência segundo causas externas do Brasil. Ministério da Saúde: Brasília, 2010. 
CAMARGO, F.C. et al. Evolução temporal dos anos potenciais de vida perdidos em óbitos por agressão. Rev. Enferm. Atenção Saúde, v. 7, n. 2, p. 68-82, 2018.

CERQUEIRA, D. et al. Atlas da violência. Brasília: Ipea, 2018.

CUNNINGHAM, R. M. et al. Violent reinjury and mortality among youth seeking emergency department care for assault-related injury: A 2-year prospective cohort study. JAMA Pediatr., v. 169, n. 1, p. 63-70, 2015.

FONSECA, V. et al. Ambiente e violência em Sergipe: mapeamento dos homicídios nos municípios sergipanos ocorridos no período 2006 a 2012. In: Fundação de Apoio à Pesquisa e à Inovação do Estado de Sergipe (Org). Pesquisa em Políticas Públicas no estado de Sergipe. Aracaju: FAPITEC, 2014.

FOWLER, K. A. et al. Childhood firearm injuries in the United States. Pediatrics, v. 140, n. 1, p. e20163486, 2017.

GRINSHTEYN, E.; HEMENWAY, D. Violent Death Rates: The US Compared with Other High-income OECD Countries, 2010. Am. J. Med., v. 129, n. 3, p. 266-273, 2016

IBGE - Instituto Brasileiro de Geografia e Estatística - Diretoria de Pesquisas, Coordenação de Trabalho e Rendimento. Pesquisa Nacional por Amostra de Domicílios Contínua, 2015. Disponível em: https://www.ibge.gov.br/estatisticas/sociais/trabalho/9171-pesquisa-nacional-por-amostrade-domicilios-continua-mensal.html. Acesso em: 5 out. 2018.

MATOS, K. F.; MARTINS, C. B. G. Perfil epidemiológico da mortalidade por causas externas em crianças, adolescentes e jovens na capital do Estado do Mato Grosso, Brasil, 2009. Epidemiol. Serv. Saúde, v. 21, n. 1, p. 43-53, 2012.

MINAYO, M. C. S. et al. Institucionalização do tema da violência no SUS: avanços e desafios. Ciên. Saúde Col., v. 23, n. 6, p. 2007-2016, 2018.

MINAYO, M. C. S. Violência contra crianças e adolescentes: questão social, questão de saúde. Rev. Bras. Saúde Mater., v. 1, n. 2, p. 91-102, 2001.

MINAYO, M. C. S. Violência, direitos humanos e saúde. In: CANESQUI, A. M. (org.). Ciências Sociais e Saúde. São Paulo: Hucitec/Abrasco, 1997.

OPAS/OMS - Organização Pan-Americana da Saúde / Organização Mundial de Saúde. Classificação estatística internacional de doenças e problemas relacionados à saúde (CID 10). Brasília: PAHO/WHO, 2018. 
ORELLANA, J. D. Y. et al. Fatores associados ao homicídio em Manaus, Amazonas, 2014. Epidemiol. Serv. Saúde, v. 26, n. 4, p. 735-746, 2017.

R CORE TEAM. R: A Language and Environment for Statistical Computing. Vienna, Austria: R Foundation for Statistical Computing, 2016.

SOURIAL, N. et al. Correspondence analysis is a useful tool to uncover the relationships among categorical variables. J. Clin. Epidemiol., v. 63, n. 6, p. 638-646, 2010.

UNICEF - United Nations Children's Fund. A familiar face: violence in the lives of children and adolescents. UNICEF: Nova Iorque, 2017. 
2 Mestre em Saúde e Ambiente; Biomédica e Enfermeira; Professora Assistente na Universidade Tiradentes - UNIT. E-mail: fernanda.fraga@souunit.com.br

3 Mestre em Saúde e Ambiente; Enfermeira; Professora Assistente na Universidade Tiradentes - UNIT.

E-mail: lourivania.oliveira@souunit.com.br

4 Mestre em Saúde e Ambiente; Médico; Professor Assistente na Universidade Tiradentes - UNIT.

E-mail: henrique.soares@souunit.com.br

5 Doutor em Medicina; Médico; Professor do Programa de Pós-Graduação em Saúde e Ambiente (PSA), Universidade Tiradentes - UNIT. E-mail: franciscopradoreis@gmail.com

6 Doutora em Medicina Clínica Cirúrgica; Médica; Professo|

A autenticidade desse artigo pode ser conferida no site https://periodicos. set.edu.br ra do Programa de Pós-Graduação em Saúde e Ambiente (PSA), Universidade Tiradentes - UNIT.

E-mail: sonia.cirurgia@gmail.com

7 Mestre em Biometria e Estatística Aplicada; Estatístico. E-mail: daniel.carvalho.ib@gmail.com

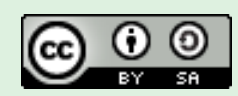

Este artigo é licenciado na modalidade acesso abertosob a Atribuição-Compartilhalgual CC BY-SA

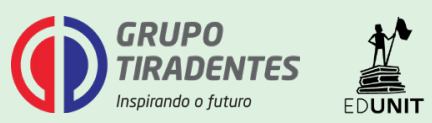

